\title{
Pesquisa de la presencia de microsporidios en conejos de la Región Metropolitana de Chile
}

\author{
CAROLINA A. MARCHANT*, SERGIO ROMERO*** y MARÍA ISABEL JERCIC** \\ SEARCH OF MICROSPORIDIOS IN RABBITS FROM \\ THE METROPOLITAN AREA OF CHILE
}

The microspore is protozoa, forced intracellular parasite, of different animal species. They belong to the Phylum Microspore, which produce very small spores and lack mitochondria. One of the microspores, there exists the Encephalitozoon cuniculi, which is an emergent opportunistic parasite, In the prevalence of $\mathbf{E}$. cuniculi in rabbits, ranks from 15 to $76 \%$ at world-wide level are reported With the purpose of detecting the presence of this parasite in our country, the search of this one was made, in the parasitology laboratory of the Institute of Public Health of Chile (ISP). The sample was constituted by 100 normal rabbits without distinction of sex, pertaining to: Bioterio of ISP, familiar deposits from Pirque, "Mundo Granja" of the University of Chile and a commercial deposit. All the rabbits were previously evaluated by means of a clinical examination. After processing the sample of deposition and tinkles, they were dyed with trichromic colorants: Cromotropo $2 R$ and Gram Cromotropo. A positive result was obtained in $55 \%$ of the individuals, where the greater percentage appears in inmunosuppressed rabbits and conventional raising rabbits. The objective of this study is to contribute to the health, in special the human being health, as well as the animals, determining the presence of $\boldsymbol{E}$. cuniculi in rabbits of different origins.

Key words: Encephalitozoon cuniculi, Microsporideos, Rabbits, Chile.

\section{INTRODUCCIÓN}

Los microsporidios son organismos unicelulares, protozoos, parásitos obligados que se caracterizan por producir esporas muy pequeñas, las que serán la forma infectante del hospedero. Se trata de microorganismos que constituyen una zoonosis, donde el reservorio principal es el conejo (Oryctolagus cuniculus), el cual elimina al parásito principalmente por la orina y deposiciones, contaminando el medio ambiente. Como el microsporidio que afecta naturalmente al conejo es Encephalitozoon cuniculi, es éste el que encontremos con mayor certeza.

La microsporidiosis es una infección humana emergente, causada por protozoos de la familia Microspora $^{2}$. Los microsporidios son organismos unicelulares, parásitos obligados de pluricelulares y se consideran eucariontes por poseer núcleo rodeado de membrana nuclear, un sistema de membranas intracitoplasmáticas y separación de cromosomas en los husos mitóticos; sin embargo, el grupo presenta algunas características comunes a los procariontes como el tamaño del rARN y la ausencia de mitocondrias, peroxisomas y membranas del aparato de Golgi ${ }^{3}$.

\footnotetext{
* Facultad de Ciencias Vetrinarias y Pecuarias de la Universidad de Chile.

** Instituto de salud Pública de Chile (ISPCh).
} 
La afinidad de las especies de microsporidios por las células hospederas es variable; algunas pueden invadir diferentes tejidos mientras otras infectan un solo tipo ${ }^{4}$. Las especies identificadas hasta ahora como parásitos del hombre son: Enterocitozoon bieneusi, E. intestinales (antes Sepata intestinalis), E. hellem y E. cuniculi².

El primer caso humano, documentado, fue informado en 1959 cuando se aisló Nosema cuniculi de la sangre, el líquido cefalorraquídeo y la orina de un niño japonés de 9 años con meningo-encefalitis febril ${ }^{4}$.

A nivel de Latinoamérica, Argentina y Cuba, se informan prevalencias entre un 50\% y $70 \%$ en explotaciones de conejos y/o bioterios, causando pérdidas económicas e interfiriendo en el desarrollo de trabajos de investigación ${ }^{5}$.

Afecta a mamíferos como conejos, ratones, hámster, cobayos, perros, gatos, zorros, visones, además de aves y al hombre ${ }^{6}$. Se estima que una posible vía de infección son los aerosoles ${ }^{7}$, por lo que se estaría frente a una transmisión de tipo horizontal, por contacto directo, sobre todo por vía aérea a través de la orina y por contacto directo con animales infectados. La transmisión vertical o transplacentaria ha sido comunicada en conejos ${ }^{8}$.

Las esporas producidas son uninucleadas, miden 2 a 4 micrómetros de largo y 1 a 1,5 de ancho y presentan 5 a 7 vueltas del tubo polar, ordenadas en una hilera ${ }^{9}$.

Después de la ingestión, el ooquiste entra al epitelio intestinal del anfitrión u hospedero, donde ocurre la primera fase de multiplicación, esta multiplicación masiva rompe la célula hospedera, con la difusión por vía sanguínea a otros órganos como hígado, pulmones, cerebro y riñones.

Presentación en el conejo: La mayoría de las infecciones parecen ser asintomáticas, pero cuando E. cuniculi afecta el sistema nervioso central, produce en el cerebro lesiones focales en forma de pequeños granulomas ampliamente diseminados, con o sin necrosis. Se manifiesta clínicamente por inclinación de la cabeza, incontinencia urinaria, paresis, temblores, convulsiones, ataxia y $\mathrm{coma}^{10}$. Las lesiones se encuentran más comúnmente en los riñones, cerebro, pulmones, bazo, glándulas suprarrenales, páncreas y miocardio ${ }^{11}$.

Presentación en el hombre: Las personas más susceptibles son los pacientes inmunocomprometidos y las transplantadas, pero es poco frecuente en inmunocompetentes. Por lo general, la infección provoca diarrea crónica en los casos de seres humanos infectados y con cuadros sistémicos, los órganos afectados principalmente son cerebro y riñones ${ }^{2}$.

Los síntomas característicos son pérdida de peso, causada por la mala absorción y diarrea acuosa, abundante, no sanguinolenta, sin mucosidades ni leucocitos ${ }^{12}$.

Respuesta inmunológica: La infección permanece en estado latente cuando la multiplicación de los parásitos es controlada por el sistema inmunológico competente del individuo.

Se ha observado en animales de experimentación que la infección con microsporidios induce la producción de anticuerpos, pero éstos al parecer no son protectores debido a que no inducen la destrucción del parásito; por el contrario, en individuos con compromiso de la inmunidad celular, la persistencia de macrófagos es un reflejo de la cronicidad de la infección. Por consiguiente, la infección por microsporidios no es exclusiva del hospedero severamente inmunocomprometido; porque cuando existe una respuesta inmune balanceada, se sugiere que los microsporidios permanecen por períodos de latencia al interior del enterocito, sin presentación de ninguna manifestación clínica ${ }^{12}$.

Métodos diagnósticos: El diagnóstico se dificulta por el tamaño diminuto de las esporas, de 1 a 4 micrones. El diagnóstico se realizaba a partir del estudio de biopsias por microscopía electrónica de transmisión; sin embargo, esta metodología tiene serias desventajas: es invasora, de alto costo y su sensibilidad es relativamente baja ${ }^{4}$.

Otras técnicas diagnósticas utilizadas son: Reacción en cadena de la polimerasa (PCR) y Western blot, entre otros, pero aún son de difícil acceso como examen de rutina para laboratorios clínicos.

Las coloraciones empleadas para la detección de microsporidios incluyen: hematoxilina-eosina, coloración de Gram, Giemsa y tinción tricrómica modificada de Weber como el Cromotropo modificado ${ }^{4}$. Todas estas coloraciones tienen un valor predictivo positivo de $100 \%$, con excepción de la hematoxilina-eosina que es de $94 \%$. La coloración tricrómica emplea Cromotropo modificado que permite la observación con microscopio de luz, las esporas se colorean de color rosado claro con un fondo verde azul, 
algunas aparecen transparentes, pero la mayoría muestran una pequeña línea central que corresponde al túbulo polar, lo que confirma el diagnóstico.

La prueba del Quit-hot utiliza además del Cromotropo la tinción de $\mathrm{Gram}^{13}$, por lo tanto, las esporas se tiñen de violeta oscuro, muestran como mínimo uno de los rasgos estructurales característicos de los microsporidios y además se observan esporas como gránulos Gram positivos.

Es necesario realizar una estimación de la presencia de este protozoo en estos animales a nivel nacional, ya que de hecho a nivel mundial, se reporta en la literatura una prevalencia que esta entre rangos muy amplios de E. cuniculi en conejos.

En Chile, no se encontraron registros de estudios detallados sobre la presencia de este parásito, ni menos de su aislamiento. Es por esto, que se recopilaron muestras de orina y deposiciones de conejos provenientes de distintos orígenes, como Bioterio y de crianza convencional, para determinar la potencial presencia del $E$. cuniculi, siendo este el objetivo de este estudio.

\section{MATERIAL Y MÉTODOS}

La muestra en estudio comprendió a 100 conejos de edades similares, menores de 1 año, sin distinción de sexo. Cincuenta animales procedían del Bioterio del Instituto de Salud Pública, 22 de Criaderos familiares de la localidad de Pirque, 8 de un Criadero comercial de la Comuna de la Pintana y 20 del "Mundo Granja”, perteneciente a la Facultad de Ciencias Veterinarias y Pecuarias de la Universidad de Chile.

Un grupo de 25 conejos procedentes del Bioterio del Instituto de Salud Pública fue sometido a un tratamiento de inmunosupresión, previo al estudio de pesquisa de E. cuniculi esperando que al disminuir la inmunidad del animal, el E. cuniculi como patógeno oportunista, apareciera en las heces y en la orina. La administración de metilprednisolona $1 \mathrm{mg} / \mathrm{kg}$ de peso vivo por vía subcutánea durante tres días consecutivos y considerando que el peso promedio de los conejos fue $3 \mathrm{~kg}$, cada animal recibió $3 \mathrm{mg}$; junto a este tratamiento a estos animales se les proporcionó tetraciclina en el agua de bebida 500 mg por animal por tres días, y así controlar la flora bacteriana oportunista.

Los restantes 75 conejos no fueron sometidos a inmunodepresión y sólo se les recolectó las muestras de orina y deposiciones. La obtención de orina y deposiciones, en el caso de animales inmunosuprimidos, se realizó una semana y media después al término del tratamiento de inmunosupresión.

Se utilizó la tinción Cromotropo 2R y la tinción con Gram Cromotropo para la visualización de presencia de esporas en las muestras de deposiciones y de orina. La observación de cada lámina se realizó en un microscopio de luz con objetivo de inmersión en aceite, con un aumento de $100 \mathrm{x}$.

Luego de realizar la tinción de las muestras se consideró como muestra positiva aquella en la que se observa al menos un grumo de microsporidios o esporas sueltas, en la muestra de orina o de deposición. La técnica de Cromotropo 2R, se observó: El tamaño de las esporas de microsporidios de 1 a $3 \mu \mathrm{m}$.

El control positivo que se utilizó corresponde a una muestra $(+)$ utilizada en la rutina del Laboratorio de Referencia de Parasitología del ISP. Las técnicas de tinción fueron facilitadas por la Sección de Parasitología del Instituto de Salud Pública, donde además se procesaron las muestras.

La selección de la muestra utilizada, fue dirigida a conejos clínicamente sanos, de distintos orígenes de la Región Metropolitana. El método estadístico utilizado en este estudio fue la de $\chi^{2}$ cuadrado para la comparación de positividad a E. cuniculi en conejos de la Región Metropolitana, según estado inmunológico y según origen de los conejos. La Prueba “exacta” de Fisher utilizada cuando el tamaño de la muestra fue pequeño. Se utilizó además la Prueba de Bonferroni cuando se comparó muchas veces una proporción.

\section{RESULTADOS Y DISCUSIÓN}

Se encontró un 55\% positivod a infección por E. cuniculi (55/100). En este porcentaje se incluyeron a los conejos sometidos a inmunosupresión, puesto que si bien estuvieron bajo un tratamiento que controlaba su estado inmunológico, su condición era de positividad. Los resultados de positividad, obtenidos al analizar las muestras 
de orina y de deposición, según la condición inmunológica de los individuos indican que el $76 \%$ de los conejos inmunosuprimidos fueron positivos a E. cuniculi (19/25), en tanto que de los inmunocompetentes este fue de 48\% (36/ 75).

El tratamiento de inmunosupresión fue realizado con la finalidad de obtener una disminución del sistema inmunológico de 25 conejos provenientes de Bioterio. Es necesario determinar que no se buscó que los animales enfermaran, sino sólo que si poseían microsporidios, éstos se multiplicaran y fueran eliminados a través de las deposiciones u orina ${ }^{12}$. Para esto se les administró un tratamiento de inmunosupresión por un período de tres días, inferior al acostumbrado de 10 días, generalmente utilizado para experiencias con Pneumocystis carinii en ratón ${ }^{14}$. Sin embargo, estos conejos inmunosuprimidos se observaron por un período de un mes luego de realizado el tratamiento, para corroborar o no la manifestación de la enfermedad.

Un factor importante, que se evidenció en este trabajo fue el estado inmunológico de los conejos, que influyó en la aparición de E. cuniculi observándose una dependencia estadísticamente significativa de la presencia de E. cuniculi con el estado inmunológico de los conejos. Esto concuerda con los resultados de otro trabajo en el cual se analizaron resultados obtenidos a partir de la inoculación experimental de E. cuniculi en ratones ${ }^{15}$.

La distribución de animales positivos a E. cuniculi, de acuerdo a las técnicas de tinción utilizadas, según el origen de las muestras, se presenta en la Tabla 1. Se pudo apreciar que el origen de la muestra influye en la presencia de $E$. cuniculi, quedando en claro mediante las pruebas estadísticas realizadas, que la única diferencia significativa correspondió entre los conejos del Bioterio inmunocomprometidos $\mathrm{v} / \mathrm{s}$ inmunocompetentes ( $p<0,005)$, mientras que no existe diferencias significativas entre criaderos de distintos orígenes. Por lo tanto, las distintas estancias de manejo y crianza de los conejos no determina la presencia del microsporidio. Es por esto que el Bioterio, no se excluye de la presencia de E. cuniculi, evidenciando que a pesar de las medidas de manejo sanitario a las que se somete el recinto, esto no basta para que la diseminación de estos parásitos se presente.

Como se ha mencionado anteriormente, el
Tabla 1. Distribución de conejos positivos al Microsporidio E. cuniculi, establecido por las técnicas de tinción realizadas en el laboratorio de Referencia de parasitología del ISP, según el origen de las muestras

\begin{tabular}{lcr}
\hline Categoría & $\begin{array}{c}\mathbf{N}^{\mathbf{0}} \text { animales } \\
\text { positivos }\end{array}$ & $\begin{array}{r}\mathbf{N}^{\mathbf{0}} \text { total de } \\
\text { la muestra }\end{array}$ \\
\hline $\begin{array}{l}\text { Bioterio ISP } \\
\text { (inmunocomprometidos) }\end{array}$ & 19 & 25 \\
$\begin{array}{l}\text { Bioterio ISP } \\
\text { (no inmunocomprometidos) }\end{array}$ & 7 & \\
Mundo Granja & 13 & 25 \\
Criadero Fliar. Pirque & 13 & 22 \\
Criadero Comercial & 3 & 8 \\
Total & 55 & 100 \\
\hline
\end{tabular}

diagnóstico de microsporidios, es muy dificultoso, aún más cuando no se tiene mayor conocimiento, como es el caso de E. cuniculi. A esto se le suma el pequeño tamaño de la espora, de 1 a 3 micrones y, por lo tanto, su difícil observación.

Las Figuras 1, 2 y 3 corresponden fotografías obtenidas de muestras positivas de deposiciones a E. cuniculi y a la utilización de Gram Cromotropo, donde se destaca el pequeño tamaño de las esporas (1 a 2 micras) que adquieren un color violeta. La visualización es posible gracias a un objetivo de $100 \mathrm{X}$.

Es necesario mencionar que las técnicas aquí utilizadas pueden complementarse con otras técnicas de laboratorio, como por ejemplo el

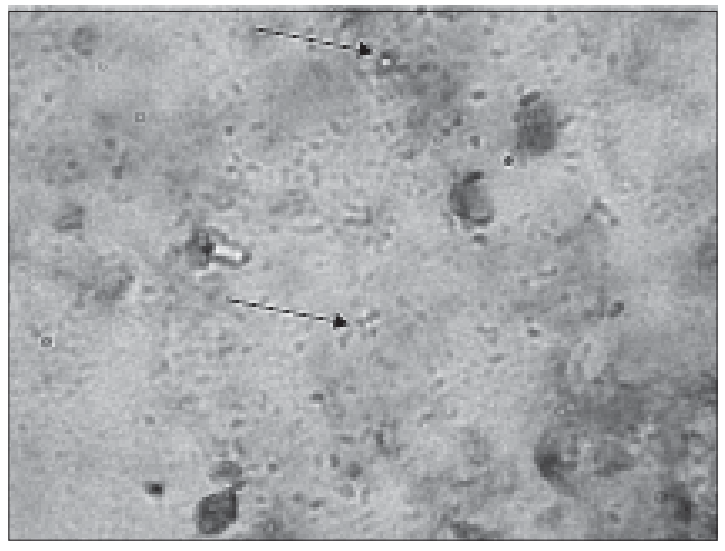

Figura 1. Esporas de E. cuniculi en muestra de deposición. Tinción Cromotropo 2R. Aumento de 100X. Cámara Digital NIKON COOLPIX 4.500. 


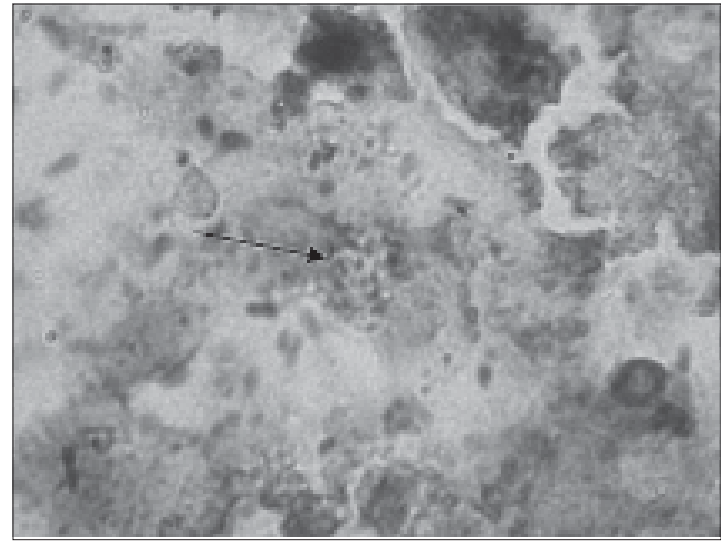

Figura 2. Esporas de E. cuniculi en muestra de deposición. Tinción Gram Cromotropo. Aumento 100X. Cámara Digital NIKON COOLPIX 4.500.

método de tinción por fluorescencia, mediante el uso de anticuerpos monoclonales específicos o mediante calcofluor ${ }^{12}$.

Se logró determinar y registrar por primera vez en Chile, mediante las técnicas de tinción tricrómicas utilizadas en laboratorio, la presencia de este parásito zoonótico, en las muestras de los conejos estudiados. La información actual de esta condición puede servir para iniciar otras investigaciones que produzcan nuevos hallazgos, con el fin de obtener un cuadro global que señale ampliamente y con más detalles, la situación que presenta nuestro país con respecto a la microsporidiosis.

\section{RESUMEN}

Se realizó un estudio para determinar la presencia y el grado de infección por Encephalitozoon cuniculi en conejos de Santiago, Chile. Se obtuvieron muestras de deposiciones y orina de 100 conejos clínicamente sanos, sin distinción de sexo, procedentes del: Bioterio de ISP, Criaderos familiares de la localidad de Pirque, "Mundo Granja” perteneciente a la Universidad de Chile y a un Criadero comercial. Luego de procesar la muestra se procedió a teñirlas con colorantes tricrómicos: Cromotropo 2R y Gram Cromotropo. Se obtuvo una positividad en un $55 \%$ de los individuos, donde el mayor porcentaje se presenta en conejos inmunosuprimidos.

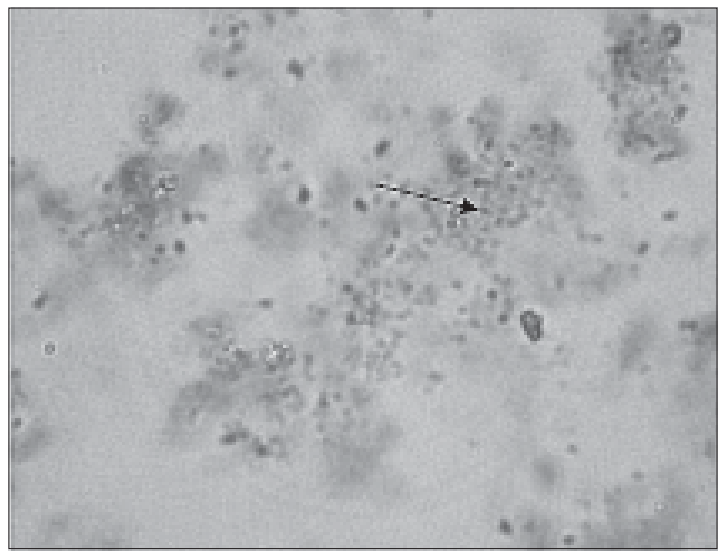

Figuras 3. Esporas de E. cuniculi en muestras de orina. Tinción Gram Cromotropo. Aumento 100 X. Cámara Digital NIKON COOLPIX 4.500.

\section{REFERENCIAS}

1.- Ansbacher L, Nichols M, Hahn A. The influence of Encephalitozoon cuniculi on neural tissue responses to implanted biomaterials in the rabbits. Lab Anim Sci 1988; 38: 689-95.

2.- Acha P, Szyfres B. Zoonosis y enfermedades transmisibles comunes al hombre y a los animales. $3^{\text {a }}$ ed. Publicación Científica y Técnica $\mathrm{N}^{\circ} 580$ OPS/ OMS. v. 3. 2003.

3.- Weber R, Bryan R, Scwartz D A, Owen R L. Human microsporidial infections. Cli Microb rev 7: 426-461. Botero, J. Montoya, M. 2002. Microsporidiosis intestinal: una visión integral. [en línea]. <http:// 66.102.7.104/search?q=cache:UiCrtJTxqjcJ: w w w. infectio.org/pdf/vol $6 / \mathrm{n} 4 /$ V6N4_2002_ARTICULO03.pdf+gram\%2 Bcromotropo\&hl=es>. [Consulta: 17-01-2006].

4.- Moncada L, Romero de Pérez G. Microsporidios en humanos. Bioméd 1998; 18: 199-215.

5.- Rodríguez B J, Cañas L, Zapata M, et al. Evaluación anatomopatológica de riñones lepóridos y determinación de la prevalencia de la encefalitozoonosis en la hacienda El Progreso de la Universidad de Antioquia. Rev Col Cienc Pec 2001; 14: 136-42.

6.- Vásquez $\mathrm{O}$, Rodríguez $\mathrm{R}$, Campos $\mathrm{T}$, et al. Microsporidiosis generalizada por Encephalitozoon sp. en un paciente pediátrico con enfermedad de Bruton. [en línea]. Bol Chil Parasitol 2001; 56: 16-21. <http:/ / www.scielo.../scielo.php? pid=s 0365 94022001000100005\&script $=$ sci_arttext\&tlng $=\mathrm{e}>$ [consulta: 22-03-2005].

7.- Weber R, Bryan R. Microsporidial infections in immunodeficient and immunocompetent patient. Clin Infec Dis 1994; 19: 517-21.

8.- Russell R J, Schilling P W. Temas Seleccionados sobre Medicina de Animales de Laboratorio: El Conejo. $3^{\text {a }}$ Ed. Centro Panamericano de Fiebre Aftosa. 1988.

9.- Cali A, Takvorian P M, Lewin S, et al. Identification of a new Nosema-like microsporidian assoicated with 
myositis in an AIDS patient. J Euk Microb 1996; 43: 108.

10.- Dykes L, Davies O. Encephalitozoon cuniculi. [en línea]. <http://www.houserabbit.co.uk/rwf/articles/ ecuniculi.html >. [consulta: 22-03-2005].

11.- Fuentealba I C, Mahoney N T, Shadduck J A, et al. Hepatic lesions in rabbits infected with Encephalitozoon cuniculi administered per rectum. [en línea]. Vet Path 1992; 29: 536-40. < http://www. vetphathology.org/cgi/conten/abstract/29/6/536 $>$ [Consulta: 22-03-2005].

12.- Botero J, Montoya M. 2002. Microsporidiosis intestinal: una visión integral. [en línea]. <http:// 66.102.7.104/search?q=cache:UiCrtJTxqjcJ : w w w. in f e c t i o.org/pdf/vol6/n 4/ V6N4_2002_ARTICULO03.pdf+gram\%2
Bcromotropo\&hl=es>. [Consulta: 17-01-2006].

13.- Moura H, Schawartz D A, Bornay-Llinares F, et al. A new and Improved «Quit-Hot gram-Chromotrope» The technique That Differentially Stains Microsporidian Sopores in Clinical Samples, Incluiding paraffinembedded tissue sections. Arch Pathol Lab Med 1997; 121: 888-93.

14.- RUDOLPH R J, VARGAS S, ROMERO S, et al. Inducción de pneumonia experimental por Pneumocistis carinii con betametasona oral. Parasitol. al Día 1996; 20: 97-9.

15.- Lallo M, Do Santos M, Fernandes E. Experimental Encephalitozoon cuniculi infection in dexamethasoneimmunosuppressed mice. Rev Sáud Públ 2002; 36: 621-6.

\section{FE DE ERRATA:}

En el título en inglés del trabajo del "Plasmodium brasilianum (Apicomplexa, PLASMODIDAE) IN THE CONGO MONKEY Alouatta brasilianum (PRIMATE: CEBIDAE) OF COSTA RICA...” que aparece en el Parasitología Latinoamericana 2006; 61: 192-196, debe de decir, tal como se indica en el título en español ...IN THE CONGO MONKEY Alouatta palliata. (PRIMATE, CEBIDAE) OF COSTA RICA

\section{Correspondencia a:}

e-mail: lcaromarchantc@gmail.com 